\title{
Using the BacMam Baculovirus System to Study Expression and Function of Recombinant Efflux Drug Transporters in Polarized Epithelial Cell Monolayers ${ }^{\mathbb{}}$
}

\author{
King Leung Fung, Khyati Kapoor, ${ }^{1}$ Jessica N. Pixley, Darrell J. Talbert, Alexandra D.T. Kwit, \\ Suresh V. Ambudkar, and Michael M. Gottesman
}

Laboratory of Cell Biology, National Institutes of Health, National Cancer Institute, Bethesda, Maryland

Received July 23, 2015; accepted November 23, 2015

\begin{abstract}
The ATP-binding cassette (ABC) transporter superfamily includes several membrane-bound proteins that are critical to drug pharmacokinetics and disposition. Pharmacologic evaluation of these proteins in vitro remains a challenge. In this study, human $A B C$ transporters were expressed in polarized epithelial cell monolayers transduced using the BacMam baculovirus gene transfer system. The purpose of the study was to evaluate the efficacy of BacMam baculovirus to transduce cells grown in monolayers. In a porcine kidney cell line, LLC-PK1 cells, baculoviral transduction is successful only via the apical side of a polarized monolayer. We observed that recombinant $A B C$ transporters were expressed on the cell surface with post-translational modification. Furthermore, sodium
\end{abstract}

butyrate played a critical role in recombinant protein expression, and preincubation in the presence of tunicamycin or thapsigargin enhanced protein expression. Cells overexpressing human P-glycoprotein (P-gp) showed vectorial basolateral-to-apical transport of $\left[{ }^{3} \mathrm{H}\right]$-paclitaxel, which could be reversed by the inhibitor tariquidar. Similarly, coexpression of human P-gp and ABCG2 in LLC-PK1 cells resulted in higher transport of mitoxantrone, which is a substrate for both transporters, than in either P-gp- or ABCG2expressing cells alone. Taken together, our results indicate that a high level of expression of efflux transporters in a polarized cell monolayer is technically feasible with the BacMam baculovirus system

\section{Introduction}

The ATP-binding cassette (ABC) superfamily is one of the largest families of proteins and is found across all organisms, from bacteria to humans. Most members of this superfamily are known to function as transporters or molecular efflux pumps using ATP as the energy source (Higgins, 1992). In humans, there are 48 known ABC transporters (Dean et al., 2001) that exhibit a wide range of substrate specificity, including nutrients, toxins, ions, and lipids. Some are known to play critical roles in cellular and biochemical processes, and their abnormal function may lead to diseases such as cystic fibrosis (e.g., ABCC7/ CFTR; Riordan et al., 1989) or phenomena such as multidrug-resistant (MDR) cancer (Szakacs et al., 2006). Since these transporters facilitate transport of their substrates against a concentration gradient, their expression is usually polarized. This feature is commonly observed in polarized cells, such as intestinal epithelial cells, capillary epithelial cells of the blood-brain barrier, renal proximal tubules cells, and

This research was supported by the Intramural Research Program of the National Institutes of Health National Cancer Institute, Center for Cancer Research.

${ }^{1}$ Current affiliation: University of California at San Francisco, San Francisco, California.

dx.doi.org/10.1124/dmd.115.066506.

S This article has supplemental material available at dmd.aspetjournals.org. hepatocytes (Shitara et al., 2006). In a polarized cell monolayer, ABC transporters are localized on the apical or basolateral layer to perform vectoral transport of the substrates.

Given the vast diversity of substrates for $\mathrm{ABC}$ transporters, these efflux pumps greatly affect drug pharmacokinetics. Therefore, many attempts have been made to develop a reliable and versatile platform in vitro to identify substrates/modulators of $\mathrm{ABC}$ transporters, measure cellular drug transport kinetics, and determine drug-drug interactions. The most commonly adopted method is the transepithelial assay, in which cell lines are allowed to form a cell monolayer in a transwell culture dish and radioactive or fluorescently labeled drugs are allowed to diffuse from either side of the membrane. The net apical-to-basal or basal-to-apical transport indicates whether the investigative compound interacts with $\mathrm{ABC}$ transporter(s). This assay can produce quantitative data on drug pharmacokinetics across a cell monolayer and therefore has become the most popular method for identification of substrates of ABC transporters (Keogh and Kunta, 2006; Volpe, 2008; Volpe, 2011; Alqahtani et al., 2013); however, the limited choice of cell lines (as they are required to have the ability to polarize), the low transfection efficiency of these cell lines, and the difficulty of maintaining transgene expression during the polarization process limit the versatility of this technique.

The BacMam virus expression system has gradually become one of the major techniques used in biochemistry, structural biology, and cell

ABBREVIATIONS: ABC, ATP-binding cassette; BAPTA-AM, 1,2-bis(2-aminophenoxy)ethane-N,N,N',N'-tetraacetic acid tetrakis(acetoxymethyl ester); DAPI, 4',6-diamidino-2-phenylindole; FACS, fluorescence-activated cell sorter; FBS, fetal bovine serum; FTC, fumitremorgin C; Glu, glutamine; IMDM, Iscove's modified Dulbecco's medium; MDR, multidrug resistance; MOI, multiplicity of infection; PCR, polymerase chain reaction; P-gp, P-glycoprotein; RT-qPCR, real-time quantitative polymerase chain reaction; TEER, transepithelial electrical resistance; THAPS, thapsigargin; TQR, tariquidar. 
biology to study gene function both in vitro and in vivo (Kost et al., 2010). Several features make this virus a good choice for recombinant gene expression. First, the BacMam virus has a broad range of host specificity. Second, it does not require a large quantity of virus for successful transduction. Third, unlike lentivirus, the BacMam virus is not able to replicate in human cells. Fourth, the BacMam virus has the capacity to carry larger fragments of DNA compared with lentivirus and adeno-associated virus. Also, it is relatively easy to generate BacMam virus, as stringent biosafety measures are not necessary. Use of the BacMam virus has certain disadvantages, however, including the high cost of scaling up production and the inability to achieve stable recombinant gene expression.

In this study, we explored the possibility of overexpressing two major $\mathrm{ABC}$ transporters (P-gp and $\mathrm{ABCG} 2$ ) on polarized cell monolayers. P-gp ( $A B C B 1 / \mathrm{MDR} 1)$ is found in human tumors and normal tissues including those of the liver, colon, and kidney, with highest expression in the adrenal gland (Fojo et al., 1987). $A B C B 1$ encodes a transmembrane protein with 1280 amino acids, with $40 \%$ sequence identity between its $\mathrm{NH} 2$ - and $\mathrm{COOH}$-terminal halves, which are connected by an 80-amino-acid linker region (Bourne et al., 1986). The importance of P-gp has been demonstrated in numerous in vitro, in vivo, and clinical studies (Shukla et al., 2011; Xia and Smith, 2012). ABCG2, also known as breast cancer resistance protein, is another major human multidrug transporter (Robey et al., 2011). Its DNA encodes a half transporter with six transmembrane domains and a nucleotide-binding domain and therefore requires homodimerization to form a functional transporter. Similar to P-gp, ABCG2 has been shown to transport a wide range of structurally and functionally diverse substrates; however, a few distinct physiologic roles of ABCG2 have been identified, including apical secretion of urate in proximal tubule cells. The failure of this transport function contributes to the occurrence of gout (Woodward et al., 2011).

To overcome cell toxicity by conventional gene transfection techniques, we chose the BacMam system for transduction. The human ABC transporters were inserted into the double-strand DNA of an engineered baculovirus. BacMam viral particles placed on the cell monolayer are absorbed into the cytoplasm by endocytosis, followed by transcription and protein expression. Our results suggest that polarized cells can be transduced using the BacMam baculovirus system and thus can be used to efficiently study the expression and function of $\mathrm{ABC}$ transporters.

\section{Materials and Methods}

Cell Lines. Cell lines were obtained from American Type Culture Collection (ATCC, Manassas, VA) and maintained under the following conditions unless specified otherwise: LLC-PK1: Medium199 (M199) + 3\% (v/v) fetal bovine serum (FBS) + 1\% penicillin/streptomycin (P/S); LLC-MDR-WT: M199+3\% $(\mathrm{v} / \mathrm{v}) \mathrm{FBS}+1 \% \mathrm{P} / \mathrm{S}+500 \mathrm{mg} / \mathrm{ml}$ geneticin; T-84: Ham's F12 medium and Dulbecco's modified Eagle's medium (1:1) $+2.5 \mathrm{mM}$ L-glutamine (L-Glu) $+5 \%$ (v/v) FBS. MA104: Eagle's minimum essential medium $+2.5 \mathrm{mM} \mathrm{Glu}+1 \% \mathrm{P} / \mathrm{S}$ + 10\% FBS; MDCK, KB-3-1, KB-V1, and Calu-3: Dulbecco's modified Eagle's medium $+2.5 \mathrm{mM} \mathrm{Glu}+1 \% \mathrm{P} / \mathrm{S}+10 \%$ (v/v) FBS; HT-29: McCoy's 5A medium + $2.5 \mathrm{mM} \mathrm{Glu}+1 \% \mathrm{P} / \mathrm{S}+10 \%(\mathrm{v} / \mathrm{v})$ FBS; Caco-2: EMEM $+2.5 \mathrm{mM}$ $\mathrm{Glu}+1 \% \mathrm{P} / \mathrm{S}+20 \%(\mathrm{v} / \mathrm{v}) \mathrm{FBS}+1 \mu \mathrm{g} / \mathrm{ml}$ vinblastine (for KB-V1). Media and antibiotics were purchased from Life Technologies (Carlsbad, CA). $\left[{ }^{3} \mathrm{H}\right]$-paclitaxel (specific activity: $60 \mathrm{Ci} / \mathrm{mmol}$ ) and $\left[{ }^{3} \mathrm{H}\right]$-mitoxantrone (specific activity: $15 \mathrm{Ci} / \mathrm{mmol}$ ) were purchased from American Radiolabeled Chemicals (St. Louis, MO). Tariquidar (TQR) was purchased from Millennium Pharmaceuticals, Inc. (Cambridge, MA). Fumitremorgin C (FMC), sodium butyrate, thapsigargin (THAPS), tunicamycin, calcein-AM, 1,2-bis(2-aminophenoxy) ethane-N, $N, N^{\prime}, N^{\prime}$-tetra-acetic acid tetrakis(acetoxymethyl ester) (BAPTA$\mathrm{AM})$, mitoxantrone, and vinblastine were purchased from Sigma-Aldrich (St. Louis, MO).
Cloning, Preparation, and Amplification of BacMam-Pg-p, BacMamABCG2, and BacMam-GFP Viruses. The expression plasmids pDest-625 containing P-gp, ABCG2, and GFP cDNA were created as described previously (Barsoum et al., 1997; Shukla et al., 2012). Briefly, the expression vectors were transformed into Escherichia coli DH10Bac cells (Invitrogen) and were cultured on selective media containing gentamicin, kanamycin, tetracycline, isopropyl $\beta$-d-thiogalactoside, and X-Gal (5-bromo-4-chloro-3-indolyl- $\beta$-d-galactoside) per the manufacturers' protocols. Transformants were selected from these plates, and bacmid DNA was generated by alkaline lysis plasmid preparation, which was further verified by polymerase chain reaction (PCR) amplification across the bacmid junctions. The plasmid and the baculovirus genomic DNA were cotransfected into Sf9 cells by Lipofectin-mediated transfection. Selected positive plaques were isolated, purified, and expanded twice in tissue culture flasks to expand viral volume. Viral titer was determined by plaque assay. The titers of the BacMam viruses, in plaque-forming units per milliliter were as follows: BacMam-Pgp $\left(1.78 \times 10^{8}\right)$, BacMam-ABCG2 $\left(7.7 \times 10^{8}\right)$, and BacMam-GFP $\left(3.47 \times 10^{8}\right)$.

BacMam Transduction. Two million cells were seeded in a transwell culture dish 6 days before transduction with $2.5 \mathrm{ml}$ of medium added to the inner chamber and $2 \mathrm{ml}$ to the outer chamber. Culture medium was refreshed every 3 days until a cell monolayer was formed. One day before transduction, medium with tunicamycin $[1 \mu \mathrm{g} / \mathrm{ml}$ (stock $5 \mathrm{mg} / \mathrm{ml}$ in dimethyl sulfoxide)] or THAPS [0.5 $\mu \mathrm{g} / \mathrm{ml}$ (stock $0.5 \mathrm{mg} / \mathrm{ml}$ in dimethyl sulfoxide)] was added to the inner and the outer chamber if necessary. Two hours before transduction, the cell monolayer was washed with medium once and then aspirated. BacMam virus (300 $\mu \mathrm{l}$ ) with a specified multiplicity of infection (MOI) was added at $37^{\circ} \mathrm{C}$ for 1 hour with gentle shaking for 15 minutes followed by the addition of medium to the outer chamber $(400 \mu \mathrm{l})$ and the inner chamber $(2400 \mu \mathrm{l})$. Two hours later, $100 \mu \mathrm{l}$ of $250 \mathrm{mM}$ freshly prepared sodium butyrate was added to both chambers (final concentration of $10 \mu \mathrm{M}$ ) and further incubated for 44 hours at $37^{\circ} \mathrm{C}$. For coexpression studies, BacMam-P-gp was added at the same time with BacMamABCG2 or BacMam-GFP using a minimal volume to yield MOI 46. The cultured cell lines used in this study had passage numbers below 10. A schematic diagram showing the experimental method is shown in Supplemental Fig. S1.

Fluorescence Microscopy. Cells grown on six-well culture plates were visualized using an EVOS FL imaging system (Life Technologies) at $40 \times$ magnification. The blue channel was used to visualize the cell nucleus (stained by DAPI) and the green channel was used to visualize GFP expressed in the cells.

Western Blot Analysis. Total protein extraction, SDS-PAGE, iBlot protein transfer, and Western blot procedures were used as previously described (Fung et al., 2014). The anti-P-gp antibody C219 (1:2000) (Agilent Technologies, Santa Clara, CA), anti-ABCG2 BXP-21 antibody (1:2000) (Abcam, Cambridge, $\mathrm{MA}$ ), and anti-Na+/K+ ATPase (1:5000) (Sigma) were used in this study.

Cell Surface Detection Using Immunolabeling and Flow Cytometry. $\mathrm{ABC}$ transporters expressed at the cell surface were detected using flow cytometry as described previously (Fung et al., 2014). Briefly, 250,000 cells were incubated in Iscove's modified Dulbecco's medium (IMDM) medium plus anti-P-gp MRK-16 monoclonal antibody (Abnova, Taipei, Taiwan) (1 $\mu \mathrm{g} /$ 100,000 cells per reaction) or anti-ABCG2 5D3-PE monoclonal antibody (eBioscience, San Diego, CA)(5 $\mu 1 / 100,000$ cells per reaction) or anti-IgG2a monoclonal antibody (1:2000) for 1 hour, after incubation with fluorescein isocyanate-labeled anti-mouse IgG2a antibody $(0.5 \mu \mathrm{g} / 100,000$ cells) (Jackson Immuno Research Laboratories, West Grove, PA). Fluorescence was recorded by a BD fluorescence-activated cell sorter (FACS) Calibur flow cytometer (BD Biosciences, San Jose, CA) using channels FL-1 (emission $488 \mathrm{~nm}$, excitation $493 \mathrm{~nm}$ ) and FL-2 (emission $488 \mathrm{~nm}$, excitation $575 \mathrm{~nm}$ ).

Drug-Accumulation Assay. Actively growing cells $\left(3 \times 10^{5}\right)$ were trypsinized and were incubated in IMDM $+5 \%$ FBS medium, with rhodamine 123 $(0.5 \mathrm{mg} / \mathrm{ml})$, at $37^{\circ} \mathrm{C}$. Cells were washed and further incubated with IMDM for 40 minutes before FACS analysis as described previously (Fung et al., 2014).

Lactate Dehydrogenase Assay. Lactate dehydrogenase activity in cells was quantitatively assayed by the CytoTox 96 Non-Radioactive Cytotoxicity Assay Kit (Promega, Madison, WI) according to the manufacturer's protocol.

Total RNA Preparation, Reverse Transcription, and Real-Time Quantitative Quantitative Polymerase Chain Reaction. Total RNA from cells was isolated using an RNAeasy Kit (Qiagen, Gaithersburg, MD). Total RNA concentration was measured by an ND-1000 spectrophotometer (NanoDrop, Wilmington, DE). Synthesis of total cDNA from $1 \mu \mathrm{g}$ total RNA reaction 
A
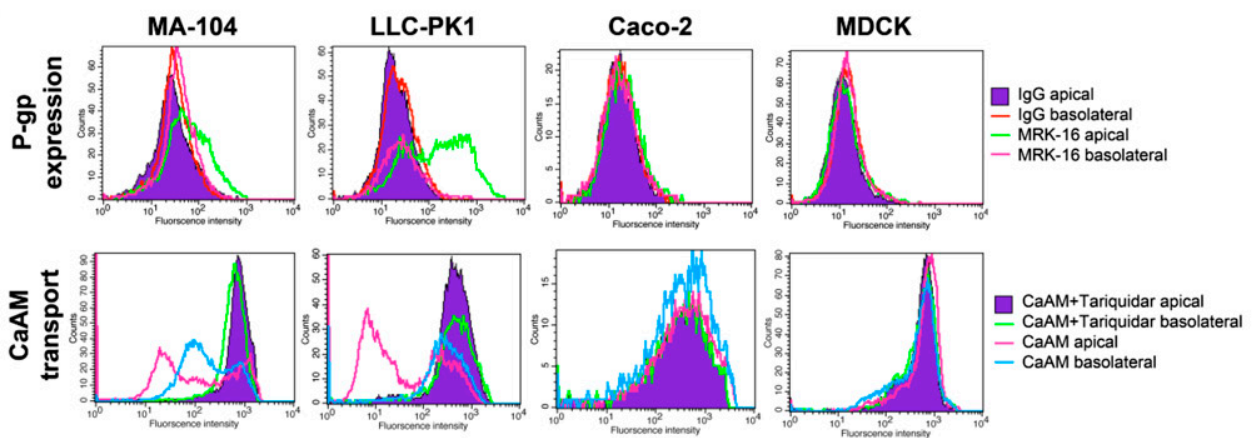

B

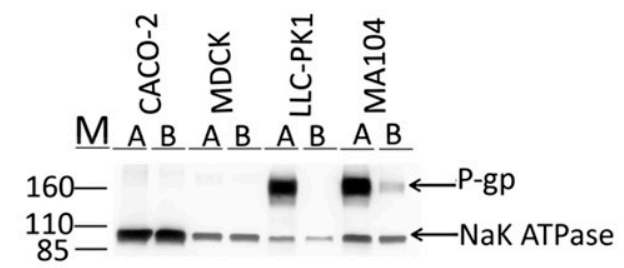

C
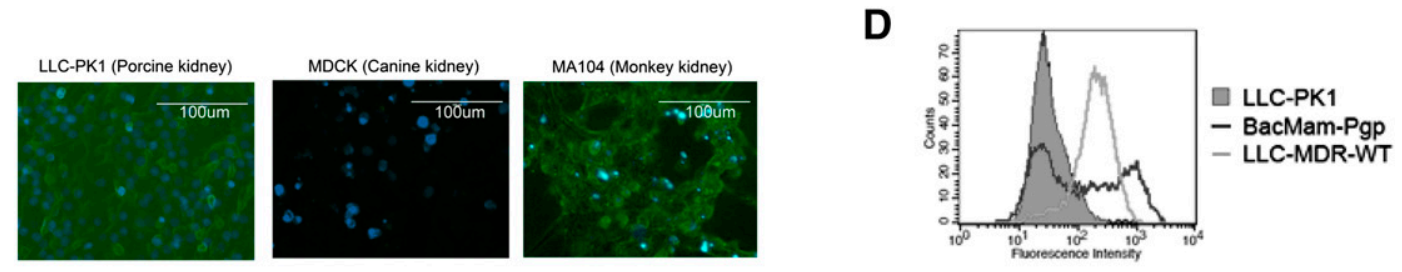

$\mathbf{E}$

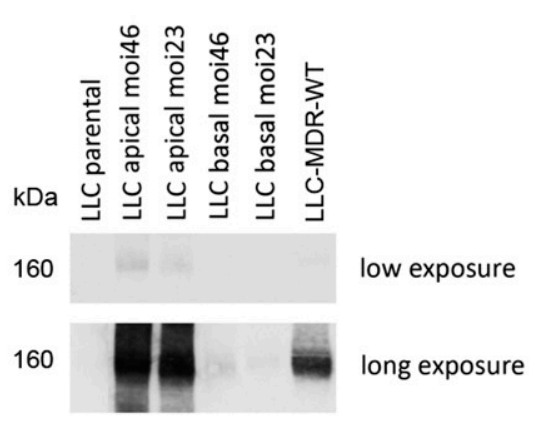

$\mathbf{F}$

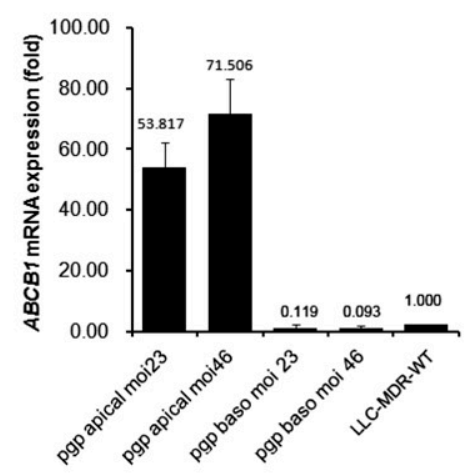

$\mathbf{G}$

\begin{tabular}{|c|c|c|c|}
\hline $\begin{array}{c}\text { Cell-line } \\
\text { name }\end{array}$ & Origin & $\begin{array}{c}\text { BacMam infection } \\
\text { via apical membrane }\end{array}$ & $\begin{array}{c}\text { BacMam infection } \\
\text { via basolateral } \\
\text { membrane }\end{array}$ \\
\hline LLC-PK1 & Porcine kidney & Yes & No \\
\hline MA104 & Monkeykidney & Yes & Yes \\
\hline T-84 & Human colon cancer & Yes & Yes \\
\hline MDCK & Canine kidney & No & No \\
\hline HT-29 & Human colon cancer & No & No \\
\hline Caco-2 & Human colon cancer & No & No \\
\hline Calu-3 & Human lung cancer & No & No \\
\hline
\end{tabular}

Fig. 1. LLC-PK1 and MA104 monolayers express human P-gp upon transduction with BacMam baculovirus. (A) Flow cytometry histograms showing recombinant P-gp expression and ability to transport calcein-AM (CaAM) in the presence and absence of TQR (1 $\mu \mathrm{M})$ in MA104, LLC-PK1, Caco-2, and MDCK cell lines. (B) Western blot analysis showing recombinant P-gp expression in Caco-2, MDCK, LLC-PK1, and MA104 cells. BacMam-Pgp virus was transduced to the polarized cells via the apical membrane $[\mathrm{A}]$ and via the basolateral membranes [B]. Na+/K+- ATPase was used as a loading control. (C) Microscopic images showing recombinant GFP expression in LLC-PK1, MDCK, and MA104 cell monolayers. Nuclear staining by DAPI (blue) in each cell line is also shown. (D) Flow cytometry histograms showing P-gp expression levels in BacMam-Pgp-transduced LLC-PK1 cells (black line) and LLC-MDR-WT cells (gray line). LLC-PK1 cells were used as a control (gray filled histogram). (E) Expression of recombinant P-gp by BacMam is influenced by BacMam virus to cell ratio. LLC-PK1 cells were transduced with BacMam virus on the apical and basolateral membranes with MOI 23 and MOI 46. Western blot analysis showing the level of P-gp expression. Short and long exposures are shown. LLC-PK1 cells and 
volume was carried out using a high-capacity cDNA kit with RNAse inhibitor (Applied Biosystems, Grand Island, NY). Relative quantification of human P-gp/ ABCB1 and glyceraldehyde-3-phosphate dehydrogenase mRNAs was measured by real-time quantitative PCR (RT-qPCR) using Hs00184491_m1 and Hs99999905_m1 probes by a 7900HT Fast Real-Time PCR System (Applied Biosystems). The RNA/cDNA preparations and RT-qPCR assays were performed according to the manufacturer's instructions. Raw data from SDS (Sequence Detection System) files were recorded and analyzed by the $2^{-\Delta \Delta \mathrm{Ct}}$ method using SDS RQ (Relative Quantification) manager software.

Transepithelial Transport Assay. Drug permeability assays were conducted in 24-mm polycarbonate transwell supports in Corning transwell culture plates (Corning, Corning, NY). Two million cells were seeded in each well, and culture medium was changed every 2 days. Cellular integrity was evaluated before and after the experiment by measuring the transepithelial electrical resistance (TEER). TEER of cell monolayers was measured using chopstick electrodes (World Precision Instruments, Sarasota, FL). Before the assay, cells were rinsed with the transport medium Hanks' balanced salt solution, $\mathrm{pH}$ 7.4, containing $10 \mathrm{mM}$ HEPES buffer. Cells were equilibrated in transport medium at $37^{\circ} \mathrm{C}$ for 30 minutes; $0.5 \mu \mathrm{Ci} / \mathrm{ml}\left[{ }^{3} \mathrm{H}\right]$-paclitaxel (specific activity: $30-90$ $\mathrm{Ci} / \mathrm{mmol}$ ) or $\left[{ }^{3} \mathrm{H}\right]$-mitoxantrone (specific activity: $15 \mathrm{Ci} / \mathrm{mmol}$ ) was applied to the donor side, and cells were incubated at $37^{\circ} \mathrm{C}$. Aliquots of sample $(100 \mu \mathrm{l})$ were taken from the receiver side at $0,120,180$, and 240 minutes and mixed with BioSafe II scintillation cocktail (Research Products International, Mount Prospect, IL). Radioactivity emitted by ${ }^{3} \mathrm{H}$, determined as disintegrations per minute, was measured using a Beckman Coulter LS-6500 liquid scintillation analyzer (Beckman Coulter, Brea, CA). The analyses were average values determined from triplicate experiments.

Calculation of Apparent Permeability Coefficients (Papp) and Efflux Ratio. The apparent permeability (Papp) was calculated according to the method previously described (Volpe, 2008). Briefly, the Papp equation is as follows: Papp $=\left(V \mathrm{r} /\left(A \times C_{0}\right)\right)(\mathrm{dC} / \mathrm{dt})$, where $V \mathrm{r}$ is the volume of the receiver chamber $\left(\mathrm{cm}^{3}\right), A$ is the transwell surface area $\left(\mathrm{cm}^{2}\right), C_{0}$ is the initial concentration of drug in the donor chamber, and $\mathrm{dC} / \mathrm{dt}$ is the initial slope of the concentration versus time. Therefore, Papp values were calculated using the slope of the steady-state rate constant $\mathrm{dC} / \mathrm{dt}$. The efflux ratios $(\mathrm{B} \rightarrow \mathrm{A} / \mathrm{A} \rightarrow \mathrm{B})$, were calculated by dividing the drug efflux rate of Papp $(\mathrm{B} \rightarrow \mathrm{A})$ by that of Papp $(\mathrm{A} \rightarrow \mathrm{B})$ to evaluate drug transporter-mediated directional efflux.

Statistical Analysis. The statistical significance of the experimental results was calculated by two-sample $t$ tests using Prism 5 (GraphPad Software Inc., La Jolla, CA). Results were considered statistically significant at $P<0.05$.

\section{Results}

The BacMam Baculovirus System Can Be Efficiently Used to Infect a Polarized Cell Monolayer. To explore the specificity of BacMam vectors on cell monolayers, we tested BacMam infection on mammalian cell lines that are able to form polarized cell monolayers in transwell plates. Cell lines were allowed to grow until polarized in the transwell plates. Cell polarization was confirmed by TEER measurement. Human cDNA was transfected onto the apical side or basolateral side of the monolayer via the BacMam vectors. We found that only a subset of cell monolayers could be productively transduced with BacMam baculovirus. After infection by BacMam-P-gp virus, a high level of P-gp protein expression was found in the LLC-PK1 and MA104 but not in MDCK or Caco-2 cells (Fig. 1, A and B). The recombinant $\mathrm{P}$-gp expressed in these cell monolayers was functional, as observed by the functional assay in transfected cells. The polarized cells LLC-PK1 and MA104 showed reduced calcein-AM accumulation, and this effect could be reversed by TQR (Fig. 1A). Using cell-surface immunolabeling and Western blot analysis, we also found that BacMam baculovirus was able to infect the apical side of the polarized LLC-PK1 cells. In MA104 cells, BacMam baculovirus infection on the apical membrane yielded higher recombinant P-gp expression and function than on the basolateral membrane (Fig. 1, A and B). A similar observation was made when we infected LLC-PK1, MA104, and MDCK monolayers with BacMam-GFP virus (Fig. 1C). BacMam baculovirus could also infect the T-84 cell line; however, the expression level of the transgene was comparatively much lower than in the LLC-PK1 or MA104 cell line (Fig. 1, B and C). When we compared the P-gp expression profiles of BacMam-P-gp with that of LLC-MDR-WT, a P-gp-expressing stable cell line, we found that the BacMam-P-gp-infected cells showed a broad range of $\mathrm{P}$-gp expression (Fig. 1D). In general, LLC-PK1 cells yielded even greater expression, and MA104 cells showed more uneven transgene expression. Using LLC-PK1 cells, we found that the P-gp protein (Fig. 1E) and P-gp mRNA (Fig. 1F) expression was dependent on the MOI of the virus, and when the MOI was between 20 and 50, high P-gp expression was observed. A table summarizing transduction specificity of BacMam virus in the cell monolayers tested in this study can be found in Fig. 1G.

Sodium Butyrate, Tunicamycin, THAPS, and BAPTA-AM Can Increase the Expression of P-gp or ABCG2 in Polarized Monolayers. Previous studies showed the importance of butyric acid in transgene expression (Condreay et al., 1999; Shukla et al., 2012). Therefore, we tested the dependence on butyrate in BacMambaculovirus-mediated transduction in polarized cells. We found that, in LLC-PK1 cells, addition of sodium butyrate $(10 \mu \mathrm{M})$ after BacMam baculoviral infection is critical for protein expression of recombinant P-gp and ABCG2 (Fig. 2, A and B). The absence of sodium butyrate renders cells unable to express recombinant protein. In addition to butyrate, preincubation of cells with tunicamycin led to a dosedependent increase of P-gp expression (Fig. 2A). Preincubation with THAPS also increased protein expression. Tunicamycin was observed to induce ABCG2 expression as well (Fig. 2B). Similarly, we found that THAPS could increase recombinant protein expression (Fig. 2, A and B). Maximal expression of both Pgp and ABCG2 was observed in the presence of $1 \mu \mathrm{g} / \mathrm{ml}$ tunicamycin or $0.5 \mu \mathrm{g} / \mathrm{ml}$ THAPS, without significant disruption of the cell monolayer (Fig. 2C) or cell death (Fig. 2D). THAPS at $0.5 \mu \mathrm{g} / \mathrm{ml}$ did not affect post-translational modification of P-gp (Fig. 2F) or transepithelial resistance (Fig. 2G); however, tunicamycin could inhibit glycosylation of P-gp, even at $0.2 \mu \mathrm{g} / \mathrm{ml}$ (Fig. 2, E and F). In addition, cell-survival assays showed that the P-gpexpressing KB-V1 cells are relatively insensitive to THAPS compared with KB-3-1 cells (Supplemental Fig. S2). Finally, we found that BAPTA-AM, a calcium chelator, could further increase recombinant ABCG2 expression (Fig. 2B), but its presence significantly decreased the monolayer resistance (Fig. 2G).

BacMam-Pgp-Transduced Cell Monolayers for Cell Permeability Analysis. To characterize the transport of P-gp substrates in BacMam-P-gp-expressing cells, we performed drug-transport assays using $\left[{ }^{3} \mathrm{H}\right]$-paclitaxel, a P-gp-specific substrate, on BacMam-P-gp monolayers and LLC-MDR-WT cell monolayers. Figure 3A shows $\left[{ }^{3} \mathrm{H}\right]$-paclitaxel transepithelial transport of polarized cells. In BacMam$\mathrm{P}$-gp-infected cells, permeability of $\left[{ }^{3} \mathrm{H}\right]$-paclitaxel from the basolateral to apical $(\mathrm{B} \rightarrow \mathrm{A})$ side is significantly higher than from the apical to basolateral side $(A \rightarrow B)$. Preincubation with TQR reverses this transport difference so that it becomes similar to BacMam-GFP-transduced cells.

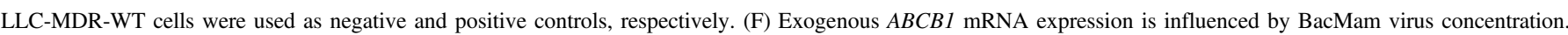

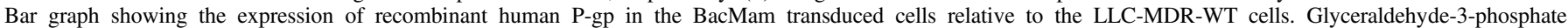
dehydrogenase was used as an endogenous gene control. (G) Table summarizing cell monolayers susceptible to transduction with BacMam baculovirus. 
A

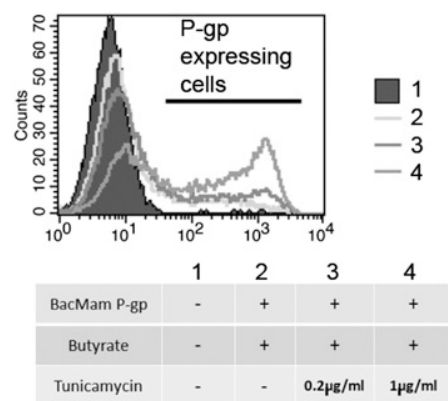

C
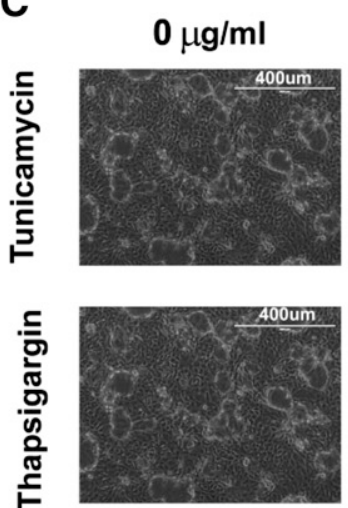

$0 \mu \mathrm{g} / \mathrm{ml}$
$0.2 \mu \mathrm{g} / \mathrm{ml}$
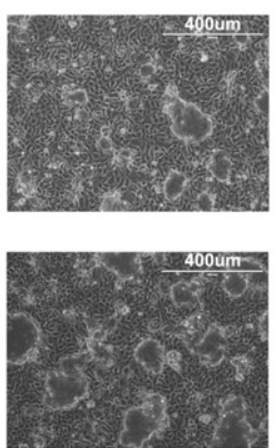

$0.1 \mu \mathrm{g} / \mathrm{ml}$

B

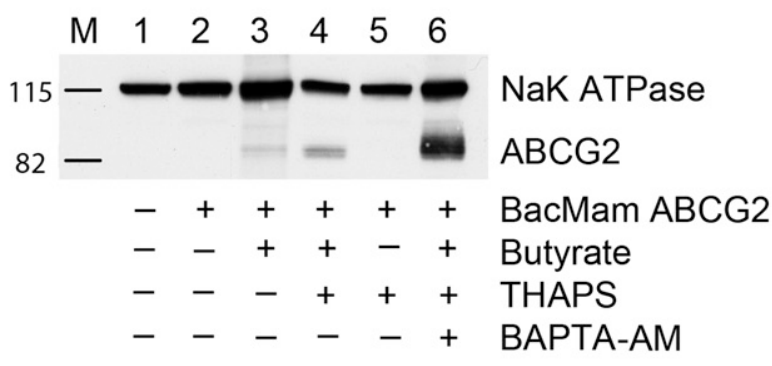

$\mathbf{D}$

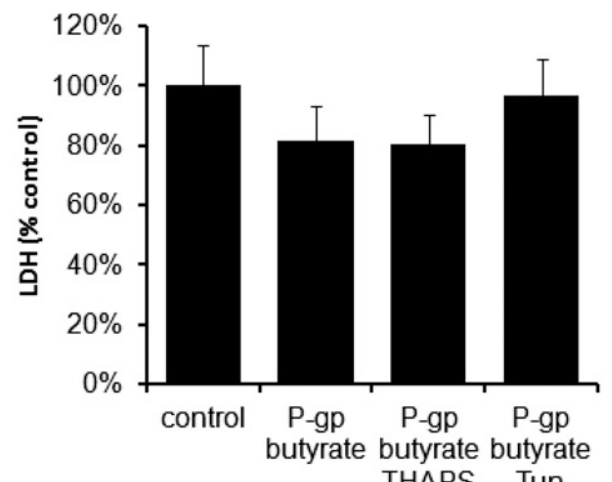

F

THAPS Tun

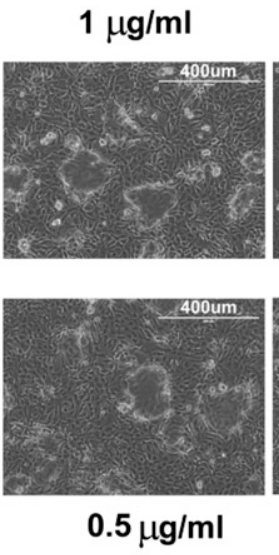

E

\section{$5 \mu \mathrm{g} / \mathrm{ml}$}
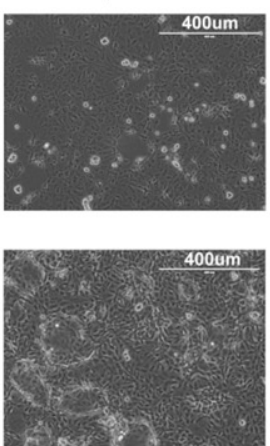

$1 \mu \mathrm{g} / \mathrm{ml}$
$20 \mu \mathrm{g} / \mathrm{ml}$
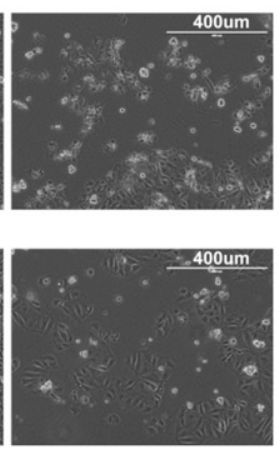

$5 \mu \mathrm{g} / \mathrm{ml}$

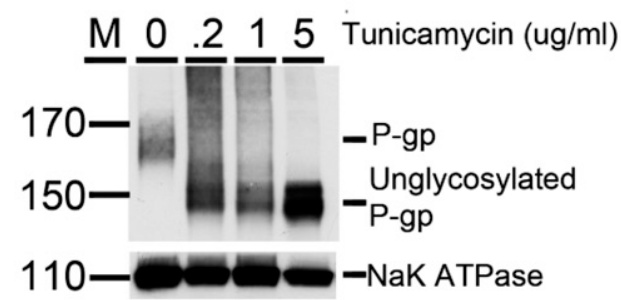

G
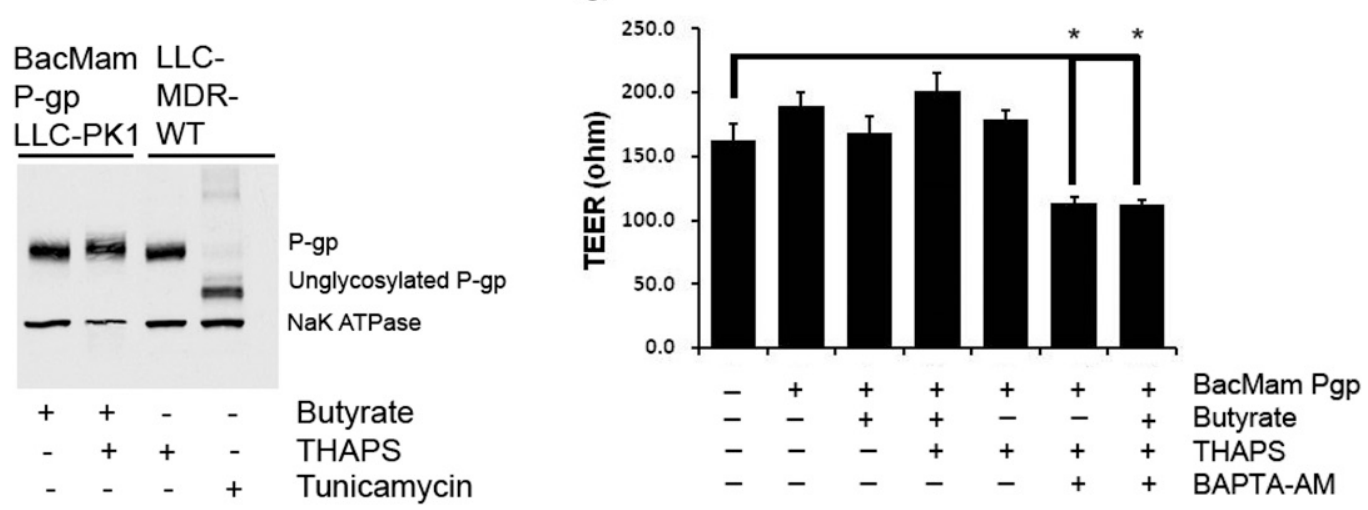

Fig. 2. Effect on recombinant P-gp or ABCG2 expression of treatment with sodium butyrate, tunicamycin, THAPS, and BAPTA-AM. (A) FACS histograms showing the effect on recombinant P-gp expression by BacMam-Pgp virus with sodium butyrate and/or tunicamycin $(0.2 \mu \mathrm{g} / \mathrm{ml}$ and $1 \mu \mathrm{g} / \mathrm{ml})$. (B) Expression of recombinant ABCG2 is dependent on sodium butyrate and can be enhanced by preincubation with THAPS $(0.5 \mu \mathrm{g} / \mathrm{ml})$ and BAPTA-AM (25 $\mu$ M). (C) Microscopic images showing the effect of tunicamycin $(0.2-20 \mu \mathrm{g} / \mathrm{ml})$ and THAPS $(0.1-5 \mu \mathrm{g} / \mathrm{ml})$ preincubation on LLC-PK1 cells. (D) Lactate dehydrogenase (LDH) activity assay showing preincubation with tunicamycin $(1 \mu \mathrm{g} / \mathrm{ml})$ and THAPS $(0.5 \mu \mathrm{g} / \mathrm{ml})$ in BacMam-P-gp-infected cells does not cause significant cell death. Polarized cells growing in transwell plates were liberated by trypsinization and assayed for LDH activity. (E) Preincubation with tunicamycin produced unglycosylated recombinant P-gp in BacMam-infected-LLC-PK1 cells in a dose-dependent manner. (F) THAPS does not affect glycosylation of P-gp. Total protein (50 $\mu \mathrm{g}$ ) from LLC-MDRWT cells treated with THAPS $(0.5 \mu \mathrm{g} / \mathrm{ml})$ or tunicamycin $(1 \mu \mathrm{g} / \mathrm{ml})$ was loaded in the same SDS-PAGE as controls. (G) Preincubation with BAPTA-AM significantly 
In contrast, the LLC-MDR-WT monolayer displayed a higher $\mathrm{B} \rightarrow \mathrm{A}$ and $\mathrm{A} \rightarrow \mathrm{B}$ drug transport ratio (13.8-fold) than the BacMam-P-gp monolayer (fivefold). In the presence of TQR, the transport ratio of LLC-MDR-WT cells and BacMam-P-gp cells is 3.7-fold to 2-fold (Fig. 3B).

Transport Function Analysis of LLC-PK1 Monolayers Coexpressing P-gp and ABCG2. Our previous study showed that ABCG2 expression was suppressed in the presence of P-gp in HeLa cells (Shukla et al., 2012), and it was suggested that expression of Pgp and ABCG2 is somewhat linked (Bark et al., 2008). Therefore, we investigated whether P-gp and breast cancer resistance protein expression is affected in the polarized cells. LLC-PK1 cell monolayers were transduced with BacMam-P-gp + BacMam-GFP, BacMam-ABCG2 + BacMam-GFP and BacMam-P-gp + BacMam-ABCG2 viruses, and transfected cells were tested for expression and function of $\mathrm{ABC}$ transporters. Immunolabeling analysis using the anti-P-gp monoclonal antibody MRK16 and the anti-ABCG2 monoclonal antibody 5D3 showed that these two transporters are colocalized on the cell surface. In contrast to HeLa cells, ABCG2 expression was not affected by P-gp in P-gp/ABCG2 coexpressing cells (Fig. 4A). Similarly, P-gp expression was not affected in either P-gp-expressing or P-gp/ABCG2coexpressing transfectants.

We further compared P-gp and ABCG2 transporter efflux function in single transporter-expressing and double transporter-expressing cells. Mitoxantrone accumulation was reduced in the P-gp- and ABCG2expressing cells. Coexpressing cells showed the lowest drug accumulation (Fig. 4B). We further examined the effect of the drug transporter's function in the presence of modulators. In this assay, we chose vinblastine, a P-gp substrate (which acts as a competitive inhibitor), and FTC, a ABCG2 modulator, as these compounds did not exhibit significant cross-inhibition. In the presence of the P-gp substrate $20 \mu \mathrm{M}$ vinblastine, which has no interaction with ABCG2, mitoxantrone accumulation was increased in P-gp-expressing cells. In the presence of $10 \mu \mathrm{M}$ FTC, ABCG2 function was inhibited. In P-gp/ ABCG2 coexpressing cells, mitoxantrone accumulation was lower when comparing single transfectants and control cells. When P-gp and ABCG2 functions were blocked by vinblastine and FTC, mitoxantrone accumulation was increased. In this experiment, we noticed that: 1) in the control experiments, mitoxantrone accumulation in the cells could be affected by vinblastine or FTC; and 2) ABCG2 function was not significantly affected in ABCG2 or P-gp/ABCG2 coexpressing cells. Finally, we conducted a transepithelial transport assay using $\left[{ }^{3} \mathrm{H}\right]-$ mitoxantrone in BacMam-P-gp and BacMam-ABCG2-transduced LLC-PK1 monolayers. In this assay, the presence of vinblastine $(20 \mu \mathrm{M})$ only marginally reduced $\left[{ }^{3} \mathrm{H}\right]$-mitoxantrone transport. Since mitoxantrone is known to be a better substrate for ABCG2 than for P-gp, $\left[{ }^{3} \mathrm{H}\right]$-mitoxantrone permeability could be effectively inhibited by (20 $\mu \mathrm{M})$ FTC (Fig. 4C). In summary, these drug accumulation studies indicate that P-gp and ABCG2 function independently in BacMamtransduced cells.

\section{Discussion}

Drug transport is an important factor that determines drug absorption and disposition. Development of in vitro models to predict drug transport and an assay for drug-drug interaction by $\mathrm{ABC}$ efflux transporters is critical for early drug development. The BacMam transfection system offers several advantages, including high efficiency, broad host specificity, portability, safety, versatility, and lack of observable cytopathic effect. In this study, we investigated the expression of recombinant $\mathrm{P}$-gp and ABCG2 in mammalian polarized cell line models for drug transport studies using the BacMam system. One of the major findings is that this system could be used to transduce several polarized cell monolayers. In particular, we found that the BacMam system could efficiently transfect ABC transporters in LLCPK1, MA104, and T84 cell lines, with LLC-PK1 cells producing the highest recombinant $\mathrm{ABC}$ transporter expression. None of the tested cell lines became resistant to BacMam baculovirus infection because of cell polarization. Unfortunately, BacMam baculovirus is unable to transduce canine MDCK and human Caco-2 cell lines, which are commonly used for drug transport assays (Volpe, 2011; Alqahtani et al., 2013).

We found that cell polarization plays a critical role in BacMam transduction. This is clearly shown by the fact that, in polarized LLCPK1 cells, only the apical, and not the basolateral, membrane is required for BacMam infection. One might argue that the virus can still attach on the basolateral side, but it is unable to transcribe recombinant genes; however, we think this scenario is unlikely since we could not find any exogenous mRNA in the BacMam-infected cells on the basolateral membrane. Cellulose-based transmembrane filters might create a physical barrier to viral attachment, which might be the cause of lower viral transduction efficiency when the virus infects the basolateral membrane. Our data suggested that the genes responsible for attachment and endocytosis of the BacMam virus are differentially expressed and are polarized in different cell lines. The exact molecular mechanism for effective transduction remains elusive, however, and warrants further investigation.

Our BacMam virus-mediated transduction method is able to express the two major $\mathrm{ABC}$ transporters (P-gp and ABCG2) in an LLC-PK1 cell monolayer. The recombinant $\mathrm{ABC}$ transporter proteins were efficiently transcribed, translated, and targeted as expected. In the LLC-PK1 cell line, P-gp and ABCG2 expression is polarized; it occurs only on the apical membrane. In fact, using liposome-mediated transfection, recombinant P-gp and ABCG2 were found only on the apical side of polarized LLC-PK1 monolayers (Takada et al., 2005; Fung et al., 2014). In addition, the transepithelial drug transport assays clearly indicate that there is increased drug efflux in the basolateral to apical direction. Therefore, we concluded that protein targeting is not affected by the BacMam baculovirus. The expression level of the recombinant protein is high enough to make the cells drug-resistant, clearly shown by the functional assays we performed. We discovered that certain ER stress inducers (tunicamycin and THAPS) could increase recombinant $\mathrm{ABC}$ transporter expression. It has been shown that these agents can induce P-gp mRNA and protein expression (Bark et al., 2008). The concentration of the compounds we used did not compromise the TEER resistance of the cell monolayer. Also, tunicamycin and THAPS do not affect protein targeting since the BacMam P-gp-transduced cells in the presence of tunicamycin or THAPS did not significantly affect $\mathrm{A} \rightarrow \mathrm{B}$ transport of $\left[{ }^{3} \mathrm{H}\right]$-paclitaxel (Supplemental Fig. S3); however, these compounds should be used cautiously, as they might affect protein maturation. For example, tunicamycin is a potent glycosylation inhibitor that can effectively impair the glycosylation of P-gp (Sereš et al., 2011). THAPS, on the other hand, is a substrate of P-gp (Supplemental Fig. S2). Its presence 
A
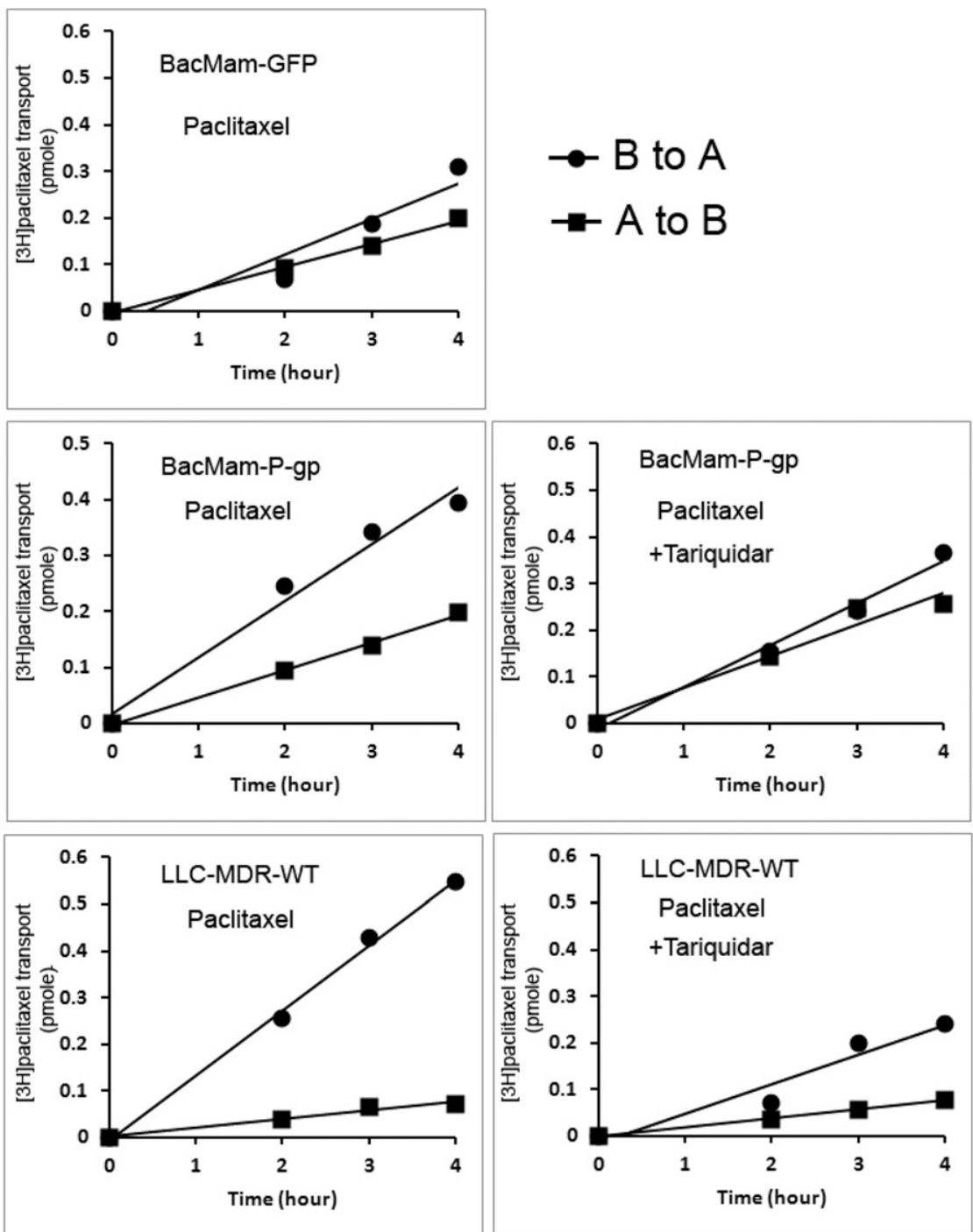

Fig. 3. Transepithelial $\left[{ }^{3} \mathrm{H}\right]$-paclitaxel transport across LLC-MDR-WT and BacMam-P-gp- or BacMam-GFP-infected LLC-PK1 cells. (A) Plots showing time-dependent increase of $\left[{ }^{3} \mathrm{H}\right]$-paclitaxel on the receiver side of the transwells with cells transduced by BacMam-GFP or BacMam-Pgp. LLC-MDR-WT cells were used to compare $\left[{ }^{3} \mathrm{H}\right]$-paclitaxel vectorial transport. To inhibit P-gp efflux activity, $1 \mu \mathrm{M}$ TQR was added 10 minutes before adding $\left[{ }^{3} \mathrm{H}\right]$-paclitaxel. Each point is the mean \pm S.D. $(n=3)$. (B) Table summarizes the fold of $\left[{ }^{3} \mathrm{H}\right]$-paclitaxel transport across LLC-PK1 cells, LLC-PK1 cells transduced with BacMam-P gp and LLC-MDR-WT cells. To inhibit P-gp function, $1 \mu \mathrm{M}$ TQR was added. Transport of $\left[{ }^{3} \mathrm{H}\right]$-paclitaxel was calculated as efflux ratio in terms of fold of drug transport $(\mathrm{B} \rightarrow \mathrm{A} / \mathrm{A} \rightarrow \mathrm{B})$ in a period of 4 hours. $* P<0.5$.

B

\begin{tabular}{ccccc} 
Sample & P-gp & ${ }^{3}$ H]Tax & $\begin{array}{c}\text { TQR } \\
(1 \mathrm{uM})\end{array}$ & $\begin{array}{c}\text { Efflux Ratio } \\
\text { (fold) }\end{array}$ \\
\hline LLC-PK1 & - & + & - & 0.70 \\
BacMam-P-gp & + & + & - & $5.0^{*}$ \\
BacMam-P-gp & + & + & + & 2.00 \\
BacMam-P-gp & + & + & - & 1.70 \\
BacMam-P-gp & + & + & + & 1.30 \\
LLC-MDR-WT & + & + & - & $13.8^{*}$ \\
LLC-MDR-WT & + & + & + & 3.70
\end{tabular}

might affect drug transport, since P-gp interacts with THAPS. Although we found the calcium chelator BAPTA-AM could enhance recombinant protein the most, compared with the other compounds tested, this compound severely affects cell polarity, which renders its use undesirable.

The drug transport assays described in this study clearly showed that BacMam baculovirus-transduced cells could express enough protein to transport $\left[{ }^{3} \mathrm{H}\right]$-paclitaxel after P-gp transfection and $\left[{ }^{3} \mathrm{H}\right]$-mitoxantrone after ABCG2 transfection. Although the transport ratio found in the BacMam P-gp-transduced cells was not as high as in the stable cell line, we suggest that this is due to the overall expression level of recombinant protein. Since the BacMam system is a transient means of transfection, it is not surprising that some of the cells in the monolayer do not express P-gp. 
A
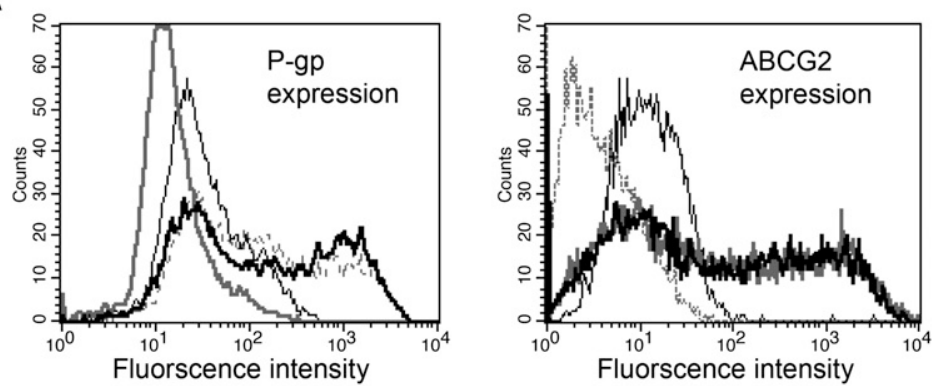

\section{Control cells \\ P-gp-expressing cells \\ ABCG2-expressing cells \\ $P$-gp and ABCG2 \\ expressing cells}

B

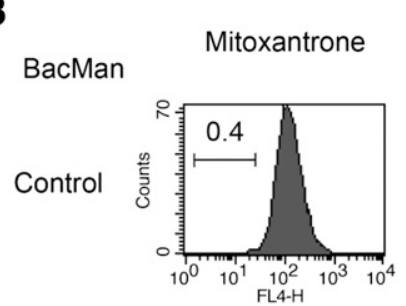

Mitoxantrone+

Mitoxantrone+ vinblastine fumitremorgin $\mathrm{C}$
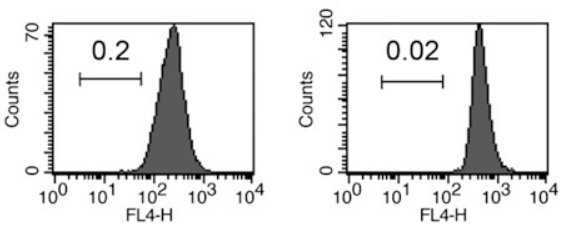

Mitoxantrone+

vinblastine+

fumitremorgin $\mathrm{C}$
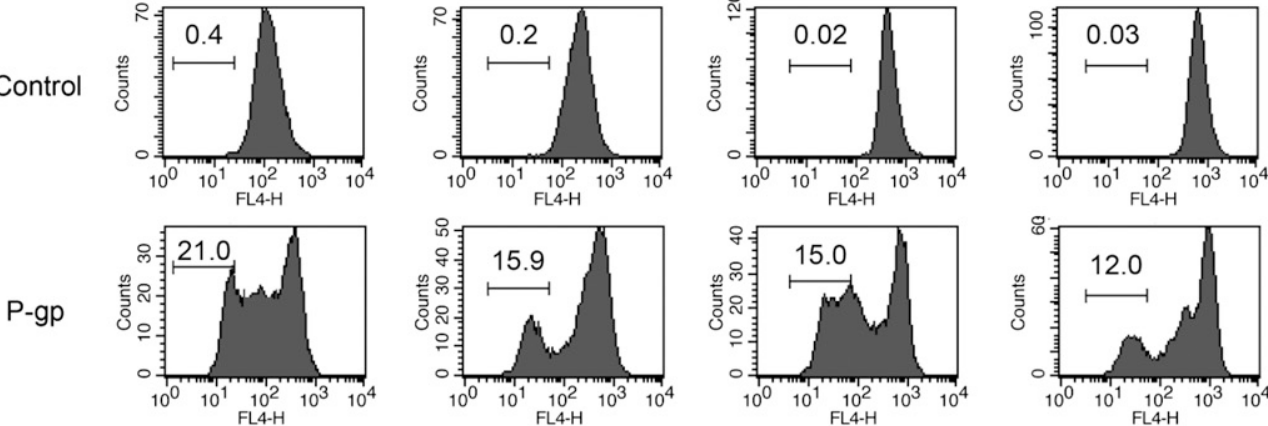

ABCG2
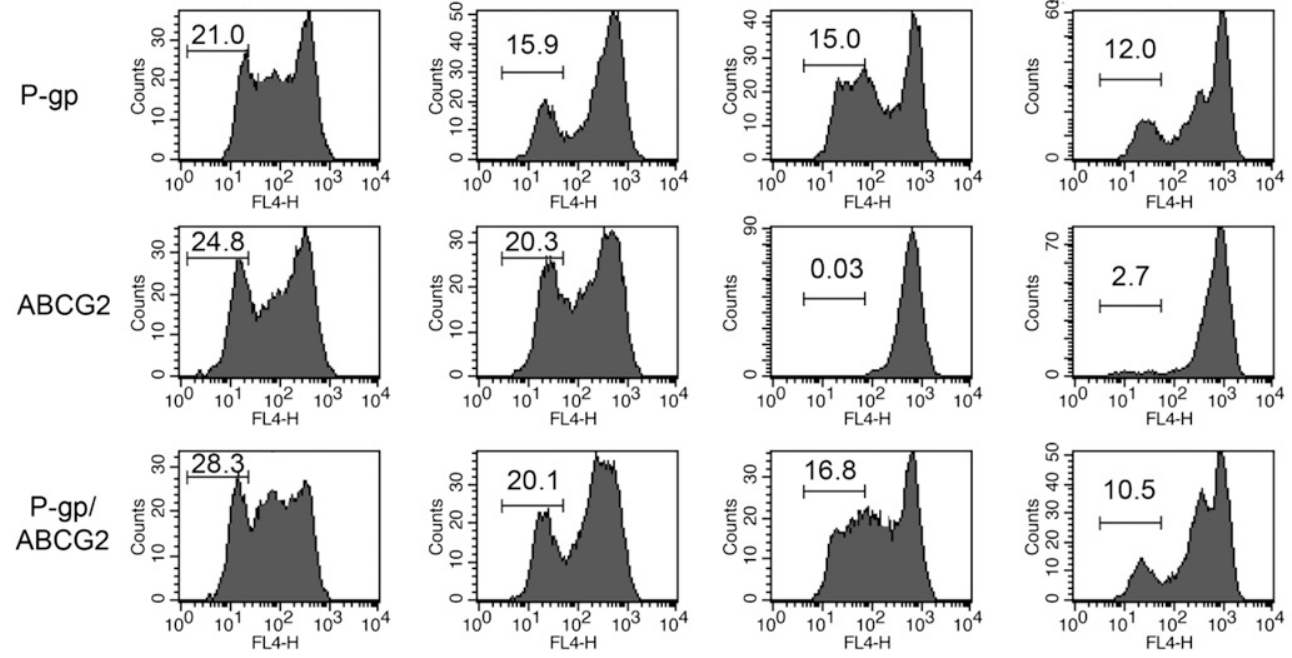

C

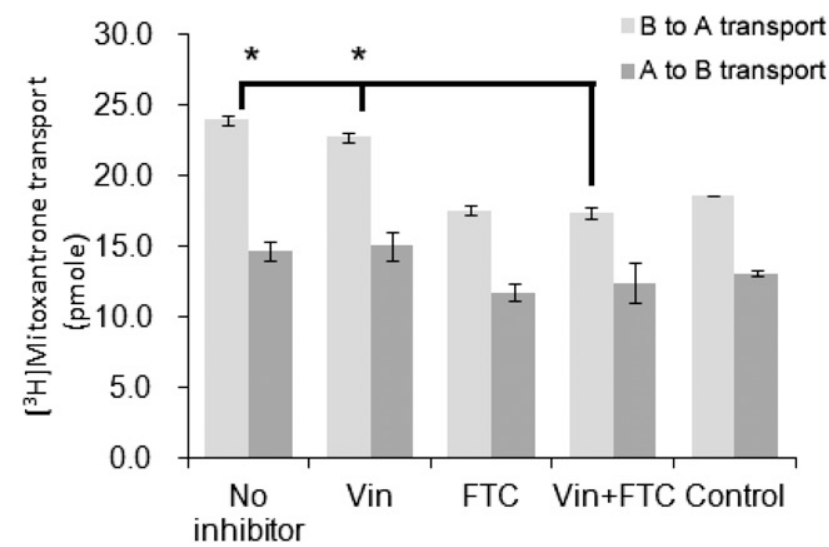

Fig. 4. $A B C G 2$ expression and function are not affected by P-gp. (A) Coexpression of recombinant ABCG2 and P-gp in LLC-PK1 cells. FACS histograms showing cell surface expression of P-gp and ABCG2 proteins in BacMam P-gp + GFP transduced cells, BacMam ABCG2 + GFP transduced cells, and BacMam P-gp + ABCG2 transduced cells. LLC-PK1 cells were used as a negative control. (B) Drug transport in P-gp and ABCG2 coexpressing cells. LLC-PK1 cells and LLC-PK1 cells coexpressing P-gp + GFP, ABCG2 + GFP, and P-gp + ABCG2 were treated with mitoxantrone. To inhibit P-gp or ABCG2 function, vinblastine ( $20 \mu \mathrm{M})$ or FTC (10 $\mu \mathrm{M})$ was added to the chambers 10 minutes before the addition of $\left[{ }^{3} \mathrm{H}\right]$-mitoxantrone. The geometric mean values of each experiment were calculated by FlowJo and are indicated in each of the histograms. (C) $\left.{ }^{3} \mathrm{H}\right]$-mitoxantrone transport across the P-gp and ABCG2 coexpressing cell monolayer. Bar chart showing $\mathrm{B} \rightarrow \mathrm{A}$ and $\mathrm{A} \rightarrow \mathrm{B}$ transport of $\left[{ }^{3} \mathrm{H}\right]$-mitoxantrone in the presence of vinblastine, FTC, and both compounds. LLC-PK1 cells transfected with BacMam virus were used as control. Each data point is the mean \pm S.D. $(n=3) . * P<0.5$. 
In this study, we used two experimental approaches to study the effect of drug transporter expression by transduction of epithelial cells with BacMam baculovirus. For flow cytometry measurements, the cells were suspended in test tubes and incubated with drugs, whereas the transepithelial assay approach requires cell polarization in the transwell chamber. The assay time required for drug transport by the flow cytometry method is shorter than the time required for the transepithelial method. We observed that drug transport kinetics of mitoxantrone by these experiments were largely similar. The transport of $\left[{ }^{3} \mathrm{H}\right]$-mitoxantrone across polarized membranes was slower than transport in a suspension of nonpolarized cells (Fig. 4, B and C). Also, vinblastine was not as effective at reducing P-gp activity in transepithelial assays, compared with cellular accumulation experiments. In any case, we showed that transepithelial transport of $\left[{ }^{3} \mathrm{H}\right]$-paclitaxel could be effectively inhibited by TQR (Fig. 3A), and in transport studies using P-gp and ABCG2 coexpressing cells, vinblastine and FTC showed maximum inhibition of mitoxantrone transport. BacMam baculovirus transduction on monolayers also provides a way to express multiple $\mathrm{ABC}$ transporters in the same host, which allows complex drug-protein or drug-drug interaction analysis. In this study, we found that recombinant ABCG2 expression and function are not affected by the presence of recombinant P-gp. Coexpression of P-gp and ABCG2 yielded similar expression levels of P-gp and ABCG2 as those of single transporter transfectants. We used cell-surface immunolabeling assays and drug transport assays to confirm these findings. These results were different from our observations in HeLa cells (Shukla et al., 2012). The exact reason for such a phenomenon is not clear, but it may be due to the nature of LLC-PK1 cells. They may lack the expression of a certain gene, such as c-Jun, which could modulate P-gp (Zhou et al., 2006) and ABCG2 (Bark et al., 2008) expression.

Overall, our results indicated that $\mathrm{ABC}$ drug transporters can be expressed in a mammalian cell monolayer using the BacMam system and exhibit expression levels high enough to perform drug permeability transport assays. We found that expression level and drug transport function of the recombinant proteins could be increased by tunicamycin, THAPS, and BAPTA-AM; however, these enhancers should be used cautiously. The experimental method we suggest in this manuscript provides a feasible approach to determine the mechanism of drug transport and the influence of $\mathrm{ABC}$ transporters on the use of various drugs.

\section{Acknowledgments}

The authors thank George Leiman for editorial assistance.

\section{Authorship Contributions}

Participated in research design: Fung, Gottesman, Ambudkar. Conducted experiments: Fung, Kwit, Kapoor, Pixley, Talbert.

Performed data analysis: Fung, Ambudkar, Kapoor.
Wrote or contributed to the writing of the manuscript: Fung, Gottesman, Ambudkar, Kapoor.

\section{References}

Alqahtani S, Mohamed LA, and Kaddoumi A (2013) Experimental models for predicting drug absorption and metabolism. Expert Opin Drug Metab Toxicol 9:1241-1254.

Bark H, Xu HD, Kim SH, Yun J, and Choi CH (2008) P-glycoprotein down-regulates expression of breast cancer resistance protein in a drug-free state. FEBS Lett 582:2595-2600.

Barsoum J, Brown R, McKee M, and Boyce FM (1997) Efficient transduction of mammalian cells by a recombinant baculovirus having the vesicular stomatitis virus $\mathrm{G}$ glycoprotein. Hum Gene Ther 8:2011-2018.

Bourne AR, Taylor JL, and Watson TG (1986) Effect of temperature on the seasonal production of testicular androgens, in vitro, by the lizard Tiliqua rugosa. Comp Biochem Physiol A 85:527-530.

Condreay JP, Witherspoon SM, Clay WC, and Kost TA (1999) Transient and stable gene expression in mammalian cells transduced with a recombinant baculovirus vector. Proc Natl Acad Sci USA 96:127-132.

Dean M, Hamon Y, and Chimini G (2001) The human ATP-binding cassette (ABC) transporter superfamily. J Lipid Res 42:1007-1017.

Fojo AT, Ueda K, Slamon DJ, Poplack DG, Gottesman MM, and Pastan I (1987) Expression of a multidrug-resistance gene in human tumors and tissues. Proc Natl Acad Sci USA 84:265-269.

Fung KL, Pan J, Ohnuma S, Lund PE, Pixley JN, Kimchi-Sarfaty C, Ambudkar SV, and Gottesman MM (2014) MDR1 synonymous polymorphisms alter transporter specificity and protein stability in a stable epithelial monolayer. Cancer Res 74:598-608

Higgins CF (1992) ABC transporters: from microorganisms to man. Annu Rev Cell Biol 8 67-113.

Keogh JP and Kunta JR (2006) Development, validation and utility of an in vitro technique for assessment of potential clinical drug-drug interactions involving P-glycoprotein. Eur J Pharm Sci 27:543-554.

Kost TA, Condreay JP, and Ames RS (2010) Baculovirus gene delivery: a flexible assay development tool. Curr Gene Ther 10:168-173.

Riordan JR, Rommens JM, Kerem B, Alon N, Rozmahel R, Grzelczak Z, Zielenski J, Lok S, Plavsic N, and Chou JL, et al. (1989) Identification of the cystic fibrosis gene: cloning and characterization of complementary DNA. Science 245:1066-1073.

Robey RW, Ierano C, Zhan Z, and Bates SE (2011) The challenge of exploiting ABCG2 in the clinic. Curr Pharm Biotechnol 12:595-608.

Sereš M, Cholujová D, Bubenčíkova T, Breier A, and Sulová Z (2011) Tunicamycin depresses P-glycoprotein glycosylation without an effect on its membrane localization and drug efflux activity in L1210 cells. Int J Mol Sci 12:7772-7784.

Shitara Y, Horie T, and Sugiyama Y (2006) Transporters as a determinant of drug clearance and tissue distribution. Eur J Pharm Sci 27:425-446.

Shukla S, Ohnuma S, and Ambudkar SV (2011) Improving cancer chemotherapy with modulators of ABC drug transporters. Curr Drug Targets 12:621-630.

Shukla S, Schwartz C, Kapoor K, Kouanda A, and Ambudkar SV (2012) Use of baculovirus BacMam vectors for expression of ABC drug transporters in mammalian cells. Drug Metab Dispos 40:304-312.

Szakács G, Paterson JK, Ludwig JA, Booth-Genthe C, and Gottesman MM (2006) Targeting multidrug resistance in cancer. Nat Rev Drug Discov 5:219-234.

Takada T, Suzuki H, and Sugiyama Y (2005) Characterization of polarized expression of point- or deletion-mutated human BCRP/ABCG2 in LLC-PK1 cells. Pharm Res 22:458-464.

Volpe DA (2008) Variability in Caco-2 and MDCK cell-based intestinal permeability assays. J Pharm Sci 97:712-725.

Volpe DA (2011) Drug-permeability and transporter assays in Caco-2 and MDCK cell lines. Future Med Chem 3:2063-2077.

Woodward OM, Köttgen A, and Köttgen M (2011) ABCG transporters and disease. FEBS J 278 : 3215-3225

Xia CQ and Smith PG (2012) Drug efflux transporters and multidrug resistance in acute leukemia: therapeutic impact and novel approaches to mediation. Mol Pharmacol 82:1008-1021.

Zhou J, Liu M, Aneja R, Chandra R, Lage H, and Joshi HC (2006) Reversal of P-glycoproteinmediated multidrug resistance in cancer cells by the c-Jun NH2-terminal kinase. Cancer Res 66: $445-452$.

Address correspondence to: Michael M. Gottesman, Laboratory of Cell Biology, 37 Convent Dr., Room 2108, National Cancer Institute, National Institutes of Health, Bethesda, MD 20892. E-mail: mgottesman@nih.gov 